\title{
Mass proportional damping in nonlinear time-history analysis
}

\author{
${ }^{*}$ Chen Xiaoming ${ }^{1 \mathrm{a}}$, Duan $\mathrm{Jin}^{1 \mathrm{~b}}$, Li Yungui ${ }^{1 \mathrm{c}}$ \\ ${ }^{1}$ China state construction technical center, Beijing, China \\ ahanee@126.com, ${ }^{b}$ Duanjin@cscec.com, ${ }^{\mathrm{C}}$ Liyungui@cscec.com
}

\begin{abstract}
Keywords: Rayleigh damping, mass proportional damping, Elastic-plastic, Time-history analysis Abstract. Rayleigh damping is the main form in direct integral method for structural nonlinear dynamic analysis under seismic wave. As stiffness proportional damping usually should be neglected for its significant influence on limit time increment, reasonable mass proportional damping becomes more important in actual engineering. A new form of mass proportional damping has been formulated in form of multi important frequencies and their mode participation coefficients instead of the simplified form which only contains the fundament frequency, and it can simulate the damping system more reasonably than the latter damping model in those complex structures.
\end{abstract}

\section{Introduction}

In the nonlinear time-history analysis for high-rise buildings, energy dissipation includes plastic dissipation, damage dissipation, damping dissipation, and so on. Compared with plasticity and damage, usually the damping is not the main source of energy dissipation in the expected rare earthquake, but improper damping model still can introduce significant error to the analysis.

In general, it is difficult to quantify the source of a system's damping and it is even more difficult to determine the value of the damping accurately. Therefore, the damping forces are usually derived by critical viscous damping $\xi$ which can be obtained by test. In the Chinese code for seismic design of buildings[1], the critical damping ratios of concrete and steel are presented, and based on the material damping, model damping method and Rayleigh damping method are widely used for simulating the damping dissipation of the whole system.

Model damping has the merit of simulating each mode accurately in a large range of frequency, so it is widely used in engineering especially for elastic analysis. For elastic-plastic analysis under strong earthquake, frequencies are not still fixed values because of the variation of material stiffness, and then the model damping method isn't still as accurate as in elastic analysis.

Compared with model damping method, Rayleigh damping can be formulated easily into the direct integration method of dynamic analysis. Though its physical demonstrations are still not plenary as proposed by E.L. Wilson[2], it is still widely used in many FEA software especially for explicit method. And for the efficiency merit of explicit method, the research on Rayleigh damping is valuable for structural analysis.

In Rayleigh damping method, as the proposal of R. Clough[3], the mass proportional damping $\alpha$ and the stiffness proportional damping $\beta$ can be solved out from two equations by the fundamental frequency and an important high frequency. Through these two parameters, it can be quantified that a low level of damping would be introduced for the zone within these selected two frequencies, but for the other frequencies, the precision of this model is still undeterminable. In explicit method, the stability limit of time increment will be reduced significantly because of the introduction of stiffness proportional damping, so it is proposed to be neglected by some software, such as ABAQUS[4]. Although those high frequencies can be damped effectively by even small stiffness proportional damping presented by X.M. Chen[5], the influence on the total response as displacement angle and base shear force is usually negligible, thus many engineers prefer to only take the mass proportional damping into account.

In this paper, reasonable mass proportional damping is researched for nonlinear dynamic analysis of high-rising buildings, and a new damping method is proposed for multi modes by introducing the mode 
participation coefficient. Based on this method of mass proportional damping, numerical example is analyzed to show the difference between the unique mode method and multi modes method.

\section{Mass proportional damping}

According to relationship of critical damping factor and Rayleigh damping:

$$
\xi_{n}=\frac{1}{2 \omega_{n}} \alpha+\frac{\omega_{n}}{2} \beta
$$

Where

$\xi_{n}$ is the critical damping factor of mode $n$;

$\omega_{n}$ is the nth frequency;

$\alpha, \beta$ are the factors of mass proportional damping and stiffness proportional damping respectively.

Assume the critical damping factors of the key frequencies $\omega_{i}$ and $\omega_{j}$ both equal to $\xi$, then the factors of $\alpha$ and $\beta$ can be written as follows:

$$
\alpha=\frac{2 \xi}{\omega_{i}+\omega_{j}} \quad \beta=\frac{2 \omega_{i} \omega_{j} \xi}{\omega_{i}+\omega_{j}}
$$

If neglect the stiffness proportional damping, then usually the mass proportional damping can be written in form of the fundamental period $\mathrm{T} 1$ as follows:

$$
\alpha=\frac{4 \pi \xi}{T_{1}}
$$

This widely used damping model is a linear function about $\mathrm{T} 1$, and the damping will decrease with the increasing of frequency, but in fact, those higher frequencies should be damped faster than those lower frequencies, thus it's a paradox.

Actually, it only can simulate the fundamental frequency accurately, and it underestimated the damping of those higher frequencies, so it is only suitable for those structures in which the fundamental frequency is dominant. Otherwise, the dynamic response may be overestimated not only for the displacement angle and base shear force but also the material damage. On the other hand, for those seismic waves which decay significantly after peak acceleration, the damping will play more important role as the reduction of external motivation, this damping form will also overestimate the damage of members.

For the reasons mentioned above, the mass proportional damping method proposed by this paper takes the mode participation coefficient into account. By take the mode participation coefficient as weight coefficient, the new form of $\alpha$ can be written as follows:

$$
\alpha=4 \pi \xi\left(\frac{c_{1}}{T_{1}}+\frac{c_{2}}{T_{2}}+\frac{c_{3}}{T_{3}}+\mathrm{L}\right)
$$

Where $T_{1}, T_{2}$ and $T_{3}$ are the important periods of the structure;

$c_{1}, c_{2}$ and $c_{3}$ are the mode participation coefficients of the periods above.

Compared with Eq.(3), the mass proportional damping model in Eq.(4) is more reasonable for simulating multi important modes. For high rising building, usually the first three order mode is enough because their total mode participation coefficient can be up to $90 \%$. But for some special structures, the mode participation coefficient of high order modes may even exceed the low order modes, thus their relative terms should be introduced into Eq.(4).

\section{Numerical example}

A frame-core-tube structure as shown in Fig.1(a) was analyzed by using the damping model proposed in this paper. The peak acceleration of seismic wave is $220 \mathrm{gal}$, and critical damping factor $\xi=0.05$. 
Usually the structures should be analyzed under three or more seismic waves. In this paper, only the results of the artificial wave are presented and the wave is shown in Fig.1(b).

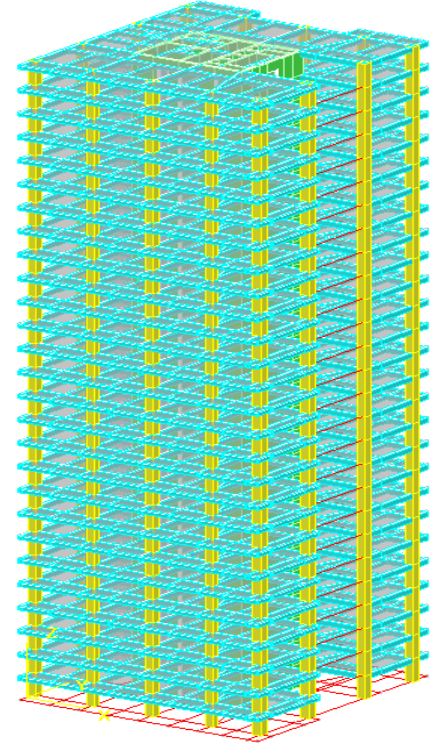

Fig.1(a) Structural model

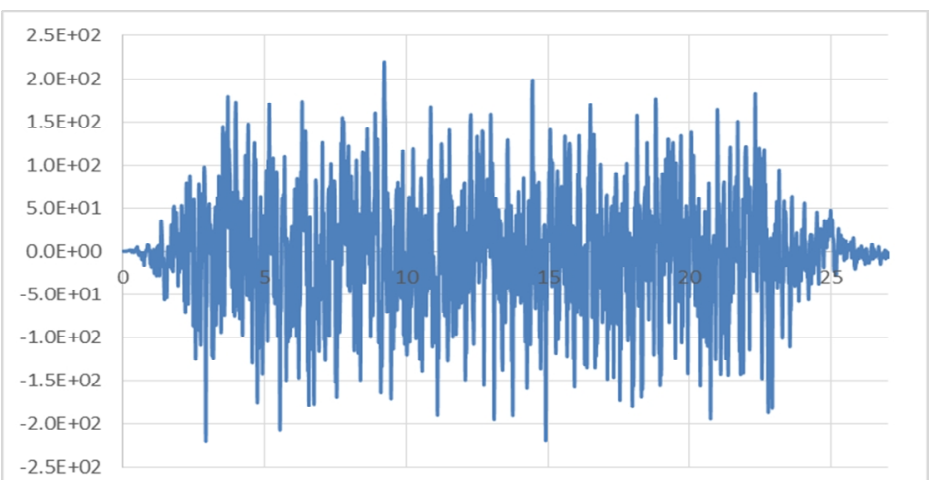

Fig.1(b) Seismic wave

For the constitutive model presented in Chinese 'Code for design of concrete structures', in which the tension skeleton curve of concrete is as follows:

$$
\begin{gathered}
\sigma=\left(1-d_{t}\right) E_{c} \varepsilon \\
d_{t}= \begin{cases}1-\rho_{t}\left[1.2-0.2 x^{5}\right] & x \leq 1 \\
1-\frac{\rho_{t}}{\alpha_{t}(x-1)^{1.7}+x} & x>1\end{cases}
\end{gathered}
$$

And the compression skeleton curve of concrete is:

$$
\begin{gathered}
\sigma=\left(1-d_{c}\right) E_{c} \varepsilon \\
d_{c}= \begin{cases}1-\frac{\rho_{c} n}{n-1+x^{n}} & x \leq 1 \\
1-\frac{\rho_{c}}{\alpha_{c}(x-1)^{2}+x} & x>1\end{cases}
\end{gathered}
$$

\begin{tabular}{|c|c|c|c|c|c|c|c|c|c|c|}
\hline \multirow{2}{*}{\multicolumn{2}{|c|}{ Period(s) }} & \multicolumn{3}{|c|}{ First order } & \multicolumn{3}{|c|}{ Second order } & \multicolumn{3}{|c|}{ Third order } \\
\hline & & 2.43 & 2.24 & 1.89 & 0.64 & 059 & 0.57 & 0.37 & 0.29 & 0.27 \\
\hline \multirow{2}{*}{$\begin{array}{c}\text { Mode } \\
\text { participatio } \\
\text { n coefficient }\end{array}$} & $\begin{array}{c}X(\%) \\
)\end{array}$ & 2.93 & 65.3 & 1.44 & 3.65 & 12.9 & 0.05 & 0.17 & 4.96 & 0.05 \\
\hline & $\begin{array}{c}\mathrm{Y}(\% \\
)\end{array}$ & 64.3 & 3.04 & 0.05 & 1.17 & 0.12 & 16.9 & 0.09 & 0.00 & 4.02 \\
\hline
\end{tabular}

The periods of elastic analysis and the relative mode participation coefficients are listed in Tab.1.

Tab.1 Results of elastic analysis

The $\alpha$ is equal to 0.26 formulated by Eq.(3), by substituting the periods and mode participation coefficients into Eq.(4), and then:

$$
\begin{array}{ll}
\alpha=0.48 & \mathrm{X} \text { direction } \\
\alpha=0.47 & \mathrm{Y} \text { direction }
\end{array}
$$

Using the final mass proportional damping $\alpha=0.47$ for dynamic analysis. An elastic model was analyzed firstly and the acceleration of seismic wave was set to zero after the peak value, then the 
structure will be changed to free vibration, so that the influence of damping to the system may be much clearer. The damping dissipations of these two damping models are shown in Fig.2, the time-histories of kinetic energy are shown in Fig.3, and the time-histories of base shear force are shown in Fig.4.

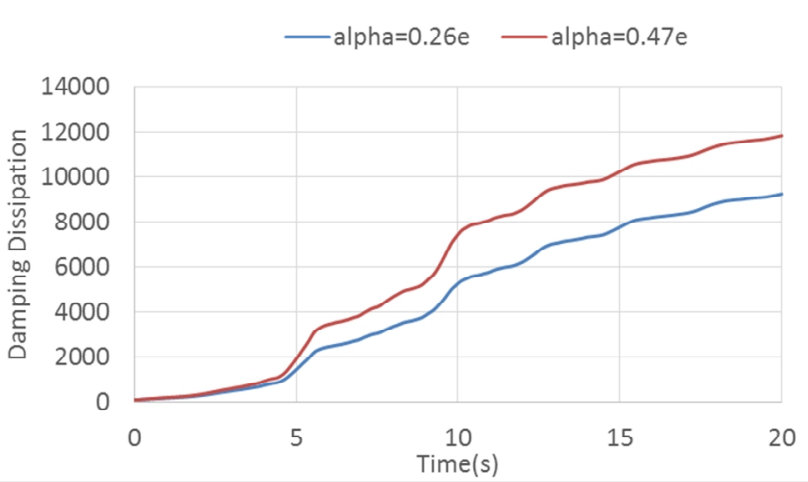

Fig. 2 Damping dissipation of elastic analysis

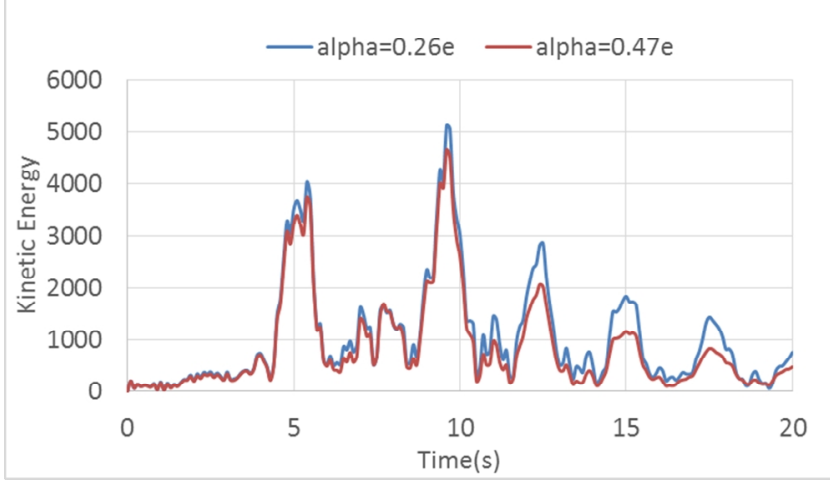

Fig.3 Kinetic energy of elastic analysis

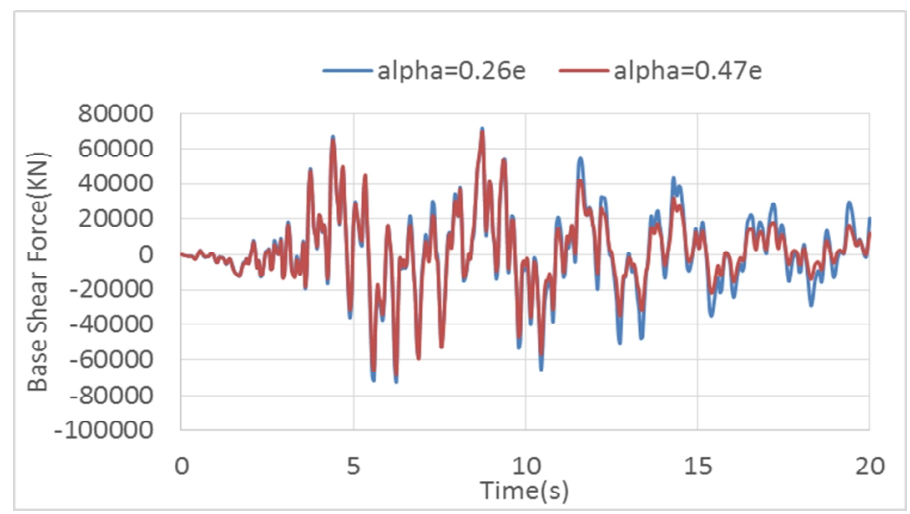

Fig.4 Base shear force of elastic analysis

As shown in Fig.2, the error of damping dissipation for these two models is about $20 \%$. When seismic wave is the dominant emotion, the difference between time-histories of both kinetic energy and base shear force is insignificant, as the seismic wave being set to zero after $10 \mathrm{~s}$, they deviated from each other. Thus it can be seen, these two damping models can lead to significant difference for those seismic waves decaying significantly.

In elastic-plastic analysis for the structure in Fig.1, difference with the elastic analysis, the seismic wave lasted about 20s. The time-histories of kinetic energy are shown in Fig.5, the damage dissipation and plastic dissipation are shown in Fig. 6 and Fig. 7 respectively, and the time-histories of base shear force are shown in Fig.8.

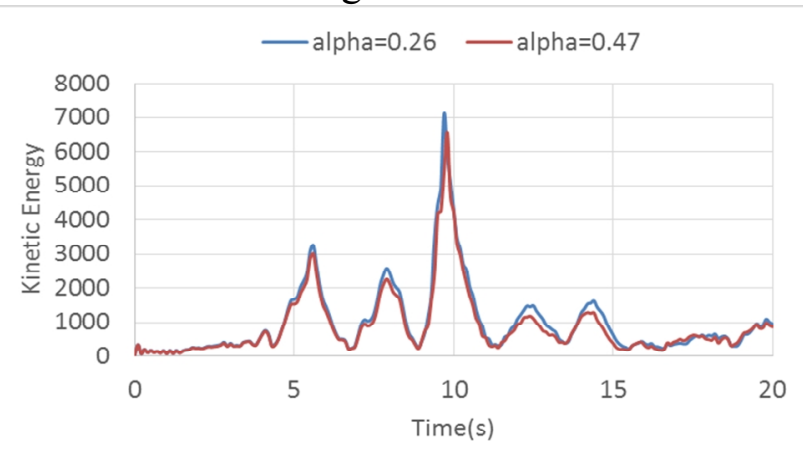

Fig.5 Kinetic energy of elastic-plastic analysis

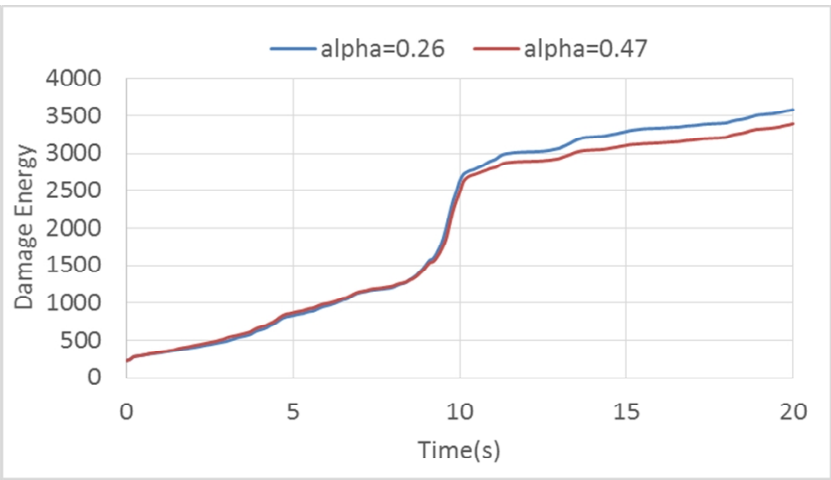

Fig.6 Damage dissipation of elastic-plastic analysis 

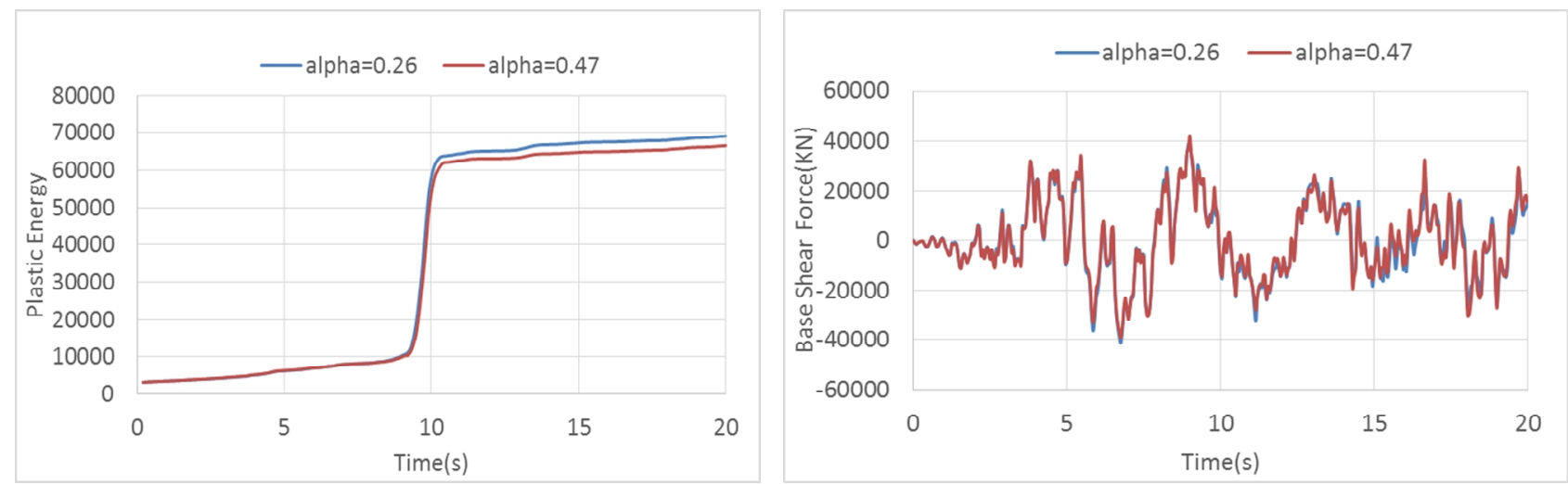

Fig.7 Plastic dissipation of elastic-plastic analysis Fig.8 Base shear force of elastic-plastic analysis

In the elastic-plastic dynamic analysis, the deviations of damage dissipation and plastic dissipation are about $5 \%$ between these two kinds of damping, and it is even more than $10 \%$ for the time-history of base shear force.

\section{SUMMARY}

Rayleigh damping is very important for the structural dynamic analysis by using explicit method. As the stiffness proportional damping usually has to be neglected for the influence on the limit stable time increment, reasonable mass proportional damping becomes much more important. Compared with the widely used model which only contains the fundamental frequency, the new model proposed by this paper can take more important frequencies into account by using the participation coefficients as the weight coefficients and then damping the vibration much more reasonably.

\section{References}

[1] Code for Seismic Design of Buildings. China architecture \& building press, Beijing, China, 2010.

[2] E.L. Wilson, Three-Dimensional Static and Dynamic Analysis of Structures: a Physical Approach with Emphasis on Earthquake Engineering. Computers and Structures, 1998.

[3] R. Clough, J. Penzien. Dynamics of Structures, McGraw Hill Kogakusha, 1975.

[4] ABAQUS User Manual. ABAQUS Inc, V6. 5. 5, 2006.

[5] X.M. Chen, J. Duan, H. Qi, and Y.G. Li. Rayleigh damping in Abaqus/Explicit dynamic analysis. Applied Mechanics and Materials, 2014, Vol. 627, p.288. 